\title{
Le système de limitation des doses
}

\author{
(Publication CIPR 26) $(*)$
}

\author{
H. P. JAMMET $(* *)$
}

\begin{abstract}
RÉSUMÉ
Dans cette analyse de la publication CIPR 26 on montre les principes qui ont abouti à l'élaboration du système de limitation des doses et en sont les conditions primordiales : la justification et l'optimisation.

L'utilisation de ces processus conduit à des normes de protection pour les travailleurs et pour les membres individuels du public en recherchant la protection la meilleure possible.
\end{abstract}

\begin{abstract}
This analysis of ICRP Publication 26 considers the basic concepts founding the development of the system of dose limitation: justification and optimisation. The use of these processes results in protection standards for workers and individual members of the public and aims at the best possible protection.
\end{abstract}

\section{INTRODUCTION}

Le système de limitation des doses de la publication 26 de la Commission Internationale de Protection Radiologique représente, sur le plan doctrinal, une étape décisive dans le domaine de la protection contre les nuisances. Cependant, il est l'aboutissement d'une longue évolution au cours des 50 années d'existence de la Commission. Le principal souci de nos prédécesseurs fut de protéger contre les effets non stochastiques par une limitation des doses. Puis la connaissance progressive des effets stochastiques cancérogènes et mutagènes conduisit à une limitation plus stricte et amorça l'introduction d'un principe complémentaire de minimisation. Les publications 1 (1959) et 6 (1962) mentionnent dans leur paragraphe 45 : Il faut souligner que les doses maximales admissibles sont des valeurs maximales; la Commission

$\left(^{*}\right)$ Exposé présenté lors de la journée S.F.R.P. sur les « Nouvelles recommandations de la C.I.P.R. ", tenue au Vésinet, le 4 juillet 1978.

$\left({ }^{* *}\right)$ Commissariat à l'énergie atomique, Institut de protection et de sûreté nucléaire, Département de protection, B.P. $\mathrm{n}^{\circ} 6,92260$ Fontenay-aux-Roses.

RADIOPROTECTION, VOL. $13-\mathrm{N}^{\circ} 4$ 
recommande de maintenir toutes les doses à des valeurs aussi faibles que possible et d'éviter toute exposition inutile. La publication 9 de 1965 reprend dans son paragraphe 52 : Comme toute exposition peut comporter un certain degré de risque, la Commission recommande d'éviter toute exposition inutile et de maintenir toutes les doses aux valeurs les plus faibles auxquelles l'on peut parvenir sans difficulté, compte tenu des aspects sociaux et économiques. Le Comité 4 devait en 1973, à la demande de la Commission, rédiger la publication 22 afin d'expliciter les principes de justification et d'optimisation contenus dans ce paragraphe 52 .

\section{LE SYSTÈME DE LIMITATION DES DOSES}

Dans sa publication CIPR 26, la Commission recommande le système de limitation des doses suivant :

a) aucune pratique ne doit être adoptée si son introduction n'entraîne pas un bénéfice net;

b) toutes les expositions doivent être maintenues au niveau le plus bas que l'on peut raisonnablement atteindre, compte tenu des facteurs économiques et sociaux;

c) l'équivalent de dose pour les individus ne doit pas dépasser les limites recommandées pour les circonstances en question.

Ces trois composantes correspondent respectivement aux principes de la justification des opérations, de l'optimisation de la protection et de la limitation des expositions individuelles.

Il faut noter que les deux principes de justification et d'optimisation constituent les conditions primordiales. Le troisième principe - celui de la limitation - est, en fait, une garantie individuelle puisque la distribution dans la population des avantages et des coûts n'est pas la même.

\section{LA JUSTIFICATION}

En principe, lorsqu'on envisage une opération ou une pratique impliquant une exposition aux rayonnements, on devrait déterminer si elle est acceptable ou non par une analyse coût-avantage, afin de s'assurer que le détriment total sera suffisamment faible par rapport aux avantages résultant de l'activité envisagée.

Dans l'analyse coût-avantage, les avantages à prendre en considération comprennent tous les avantages recueillis par la Société, qu'ils soient quantifiables ou non. De même, on considère que les coûts comprennent la somme totale de tous les aspects négatifs d'une opération, y compris les coûts monétaires et tout dommage pour la santé de l'homme ou pour l'environnement.

Pour la justification, l'analyse coût-avantage est globale et correspond à la formule $B=V-(P+X+Y)$ où $B$ est le bénéfice net, $V$ le bénéfice brut, $P$ le coût de production de base, $X$ le coût d'un niveau déterminé de 
protection, et $Y$ le coût du détriment lié à cette opération. Le coût dans ce contexte inclut les coûts sociaux et économiques.

D’autre part, il faudrait procéder à une comparaison des pratiques après optimisation et le choix final dépendra de nombreux facteurs, dont certains seulement sont liés à la radioprotection.

\section{L'OPTIMISATION}

L'optimisation, par ailleurs, est un élément essentiel de la radioprotection appliquée. Afin de déterminer si l'on peut " raisonnablement » réduire une exposition, il convient de considérer l'augmentation des avantages que procure une telle réduction et l'augmentation des coûts qu'elle implique. Pour l'optimisation, l'analyse coût-avantage est différentielle et correspond à une analyse coût-efficacité. Dans la formule utilisée dans l'analyse coût-avantage pour maximiser le bénéfice net, la variable indépendante est l'équivalent de dose collectif, $S$, résultant de l'opération. Le bénéfice net optimal serait atteint pour

$$
\frac{d V}{d S}-\left(\frac{d P}{d S}+\frac{d X}{d S}+\frac{d Y}{d S}\right)=0 .
$$

Comme on peut considérer $V$ et $P$ comme constants avec $S$ pour une pratique donnée, il s'ensuit que la condition d'optimisation est

$$
\frac{d X}{d S}=-\frac{d Y}{d S}
$$

Les évaluations basées sur l'équation ci-dessus peuvent être facilitées en assignant une valeur monétaire à l'unité d'équivalent de dose collectif. L'utilisation de l'analyse coût-avantage pour la justification et l'optimisation n'assure pas toujours une protection suffisante des individus lorsque les avantages et les coûts ne sont pas distribués de façon identique dans la population, et il devient alors nécessaire de limiter les expositions individuelles.

\section{LA LIMITATION}

La limitation des expositions vient compléter l'utilisation des processus de justification et d'optimisation. Les limites d'équivalent de dose sont destinées à prévenir les effets non stochastiques et à limiter à un niveau acceptable l'apparition des effets stochastiques.

Les précédentes limites d'équivalent de dose recommandées par la Commission ont été en vigueur pendant plus de 20 ans. Rien n'indique que cette limitation des doses n'ait pas réussi à assurer un niveau approprié de 
sécurité. Toutefois, la Commission a jugé bon de revoir ses limites d'équivalent de dose à la lumière des connaissances actuelles.

Les nouvelles limites d'équivalent de dose sont fondées sur le risque total lié à l'irradiation de tous les tissus exposés. En principe, les recommandations de la Commission devraient assurer que le détriment résultant de toute une année de pratique soit limité à une valeur indépendante de la distribution de l'équivalent de dose dans l'organisme. Les limites d'équivalent de dose doivent donc s'appliquer à l'équivalent de dose engagé résultant d'un an de pratique.

La Commission pense que, lorsqu'on évalue les risques afin de fixer des limites d'équivalent de dose pour les individus, le détriment héréditaire qui peut apparaître dans les deux premières générations après l'irradiation devrait être ajouté au détriment somatique total. Cependant, dans toute évaluation de l'ensemble des effets héréditaires, il faudra tenir compte du dommage subi par toutes les générations ultérieures. Les recommandations de la Commission ont pour but de limiter les effets somatiques pour les individus, les effets héréditaires dans la descendance immédiate des personnes irradiées, et les effets héréditaires et somatiques dans la population dans son ensemble. En ce qui concerne les individus, les limites d'équivalent de dose s'appliquent à deux catégories d'expositions, à savoir l'irradiation professionnelle pour les travailleurs et l'irradiation générale pour le public.

\section{LES LIMITES D'ÉQUIVALENT DE DOSE POUR LES TRAVAILLEURS}

La Commission estime qu'une bonne méthode pour juger de l'acceptabilité du niveau de risque dans le travail sous rayonnements consiste à comparer ce risque à celui d'autres occupations reconnues comme atteignant un haut niveau de sécurité, avec une mortalité annuelle moyenne due aux risques professionnels égale à $10^{-4}$. En première approximation, une évaluation fondée seulement sur le critère de mortalité peut être considérée comme prudente, puisque les effets non mortels de l'irradiation sont bien moins fréquents que dans d'autres professions sûres.

Le niveau de risque représentatif d'une profession sûre est le risque moyen pour tous les travailleurs dans cette branche, le risque individuel variant avec leur tâche et sa distribution tournant autour de cette moyenne. Les limites d'équivalent de dose ont pour but d'assurer une protection adéquate des individus les plus exposés. Dans des groupes importants de travailleurs, la distribution des équivalents de dose est habituellement une fonction log-normale, avec très peu de valeurs approchant les limites et une moyenne arithmétique égale au dixième environ de ces limites.

Il est peu probable que des travailleurs dont l'exposition est proche des limites d'équivalent de dose reçoivent de telles doses chaque année au cours de leur vie professionnelle et on peut s'attendre à ce que l'équivalent de dose pour toute la durée de leur vie soit nettement inférieur à celui que l'on pourrait déduire de la limite annuelle. Une exposition de longue durée 
d'une proportion considérable de travailleurs à un niveau proche des limites d'équivalent de dose ne pourrait être acceptable que dans la mesure où une analyse coût-avantages approfondie montrerait que l'augmentation de risque qui en résulte serait justifiée.

La Commission estime qu'on préviendra les effets non stochastiques en appliquant une limite d'équivalent de dose de $0,5 \mathrm{~Sv}$ par an à tous les tissus sauf le cristallin pour lequel la limite est de $0,3 \mathrm{~Sv}$ par an. Ces limites s'appliquent, que l'exposition concerne un ou plusieurs tissus.

Pour les effets stochastiques, les limites d'équivalent de dose sont fondées sur le principe que le risque doit être égal, que l'irradiation de l'organisme entier soit homogène ou non. Cette condition sera satisfaite si :

$$
\Sigma_{T} w_{T} H_{T} \leqq H_{0 e, L},
$$

où $w_{T}$ est un facteur de pondération représentant la proportion de risque stochastique lié au tissu $T$ par rapport au risque total dans le cas d'irradiation homogène, $H_{T}$ est l'équivalent de dose annuel dans le tissu $T, H_{0 e, L}$ est la limite de l'équivalent de dose annuel pour une irradiation homogène de l'organisme entier, c'est-à-dire $50 \mathrm{mSv}$.

Les valeurs de $w_{T}$ sont les suivantes : gonades 0,25 ; sein 0,15 ; moelle osseuse rouge 0,12 ; poumon 0,12 ; thyroïde 0,03 ; surfaces osseuses 0,03 ; reste de l'organisme 0,30 (avec 0,06 pour chacun des cinq tissus recevant l'équivalent de dose le plus élevé et zéro pour tous les autres tissus).

Pour l'irradiation externe par des rayonnements pénétrants, la limite annuelle de l'indice d'équivalent de dose profond dans une sphère de $30 \mathrm{~cm}$, $H_{I, p}$, est de $50 \mathrm{mSv}$.

Pour l'irradiation interne due à l'incorporation de radionucléides, la protection est basée sur des limites annuelles d'incorporation calculées par le Comité 2 de la C.I.P.R.

En cas d'irradiation externe et interne associées, la limitation des doses est donnée par la formule :

où

$$
\frac{H_{I, p}}{H_{0 e, L}}+\Sigma_{j} \frac{I_{j}}{I_{j, L}} \leqq 1,
$$

$H_{I, p}$ est l'indice d'équivalent de dose annuel,

$H_{0 e, L}$ la limite annuelle d'équivalent de dose,

$I_{j}$ l'incorporation annuelle de radionucléide $j$,

$I_{j, L}$ la limite annuelle d'ircorporation du radionucléide $j$.

Ces valeurs conviennent pour tous les travailleurs, quel que soit l'âge ou le sexe. Toutefois, des limitations spéciales de dose peuvent être nécessaires pour limiter l'irradiation de l'embryon ou du fæus lors de l'exposition professionnelle de femmes en état de procréer ou de femmes enceintes.

voL. $13-\mathrm{N}^{\circ} 4$ 


\section{LES LIMITES D'ÉQUIVALENT DE DOSE POUR LES MEMBRES INDIVIDUELS DU PUBLIC}

Les risques dus aux rayonnements ne représentent qu'une très faible fraction de l'ensemble des dangers auxquels sont exposés les membres du public du fait de leur environnement. Il semble raisonnable de considérer l'importance des risques entraînés par les rayonnements pour le grand public à la lumière de l'acceptation des autres risques de la vie quotidienne. L'analyse des informations dont on dispose sur les risques couramment acceptés dans la vie de tous les jours, montre que le niveau d'acceptabilité des risques mortels pour le public est inférieur d'un ordre de grandeur au niveau d'acceptabilité des risques professionnels. Sur cette base, un risque de l'ordre de $10^{-6}$ à $10^{-5}$ par an serait probablement acceptable pour tout membre du public.

La Commission recommande une limite d'équivalent de dose pour l'organisme entier de $5 \mathrm{mSv}$ par an pour les membres individuels des groupes de population critiques. En cas d'exposition de l'organisme entier pendant toute la vie, l'hypothèse d'un risque total de $10^{-2} \mathrm{~Sv}^{-1}$ impliquerait qu'on restreigne la limite d'équivalent de dose à $1 \mathrm{mSv}$ par an. L'application aux membres individuels du public d'une limite annuelle d'équivalent de dose de $5 \mathrm{mSv}$ conduira probablement à des équivalents de dose moyens inférieurs à $0,5 \mathrm{mSv}$. $\mathrm{Si}$, dans l'avenir, l'exposition combinée résultant d'expositions optimisées devait conduire à des équivalents de dose moyens supérieurs à $1 \mathrm{mSv}$ par an, la situation pourrait encore se justifier.

Pour les expositions non homogènes entraînant des doses $H_{T}$ aux organes, la condition de limitation est $\Sigma_{T} w_{T} H_{T} \leqq H^{\prime}{ }_{0 e, L}$, où $H^{\prime}{ }_{0 e, L}$ est la limite d'équivalent de dose pour l'irradiation homogène de l'organisme entier pour une personne du public. Afin de prévenir les risques non stochastiques, il convient d'appliquer, pour tout organe ou tissu, une limite impérative d'équivalent de dose annuelle de $50 \mathrm{mSv}$.

Ces limites pour les individus du public sont les mêmes pour les deux sexes. Mais, lors du calcul de l'équivalent de dose dû à l'incorporation de radionucléides, il faut tenir compte des différences dans les dimensions des organes et les caractéristiques métaboliques qui existent chez les enfants.

\section{EXPOSITION DE LA POPULATION}

La Commission ne propose pas de limites de dose pour les populations. Il est clair que la limite pour l'irradiation d'une population dans son ensemble est le total résultant de la sommation des contributions minimales nécessaires et non un total admissible qu'on pourrait répartir.

Toute contribution artificielle à l'exposition de la population doit être justifiée par les avantages qu'elle présente et la protection du public doit être optimisée. Le respect de ces exigences garantira vraisemblablement que l'équivalent de dose moyen pour la population n'excédera pas $0,5 \mathrm{mSv}$ par an. 


\section{NORMES DE PROTECTION}

Le système de limitation des doses aboutit à des normes de protection. Il est important de faire une distinction entre différents types de normes de protection : les limites et les niveaux.

Les limites fondamentales sont constituées par les limites primaires et secondaires.

Les limites primaires sont les limites d'équivalent de dose pour l'organisme entier et les différents organes ou tissus.

Des limites secondaires sont données pour l'irradiation externe et pour l'irradiation interne. Dans le cas d'irradiation externe de l'organisme entier, la limite secondaire s'applique à l'équivalent de dose maximal dans l'organisme à plus de $1 \mathrm{~cm}$ de profondeur. Dans le cas d'irradiation interne, les limites secondaires sont les limites annuelles d'incorporation par inhalation ou par ingestion.

Les limites dérivées sont associées à des quantités autres que l'équivalent de dose ou l'incorporation; elles sont reliées aux limites fondamentales par un modèle défini de la situation dans le but de refléter les limites fondamentales. On peut fixer des limites dérivées pour des quantités telles que le débit d'équivalent de dose à un poste de travail, la contamination de l'air, de l'eau, des surfaces...

Les limites autorisées sont des limites fixées par une autorité compétente ou par la direction d'un établissement. Ces limites, en général, seront inférieures aux limites dérivées. Le processus de minimisation est fréquemment employé lors de la fixation des limites autorisées, mais on peut également utiliser le processus d'optimisation.

Les niveaux de référence ne sont pas des limites : on les utilise pour déterminer la conduite à tenir lorsqu'une quantité dépasse ou risque de dépasser le niveau de référence; l'action à entreprendre peut aller du simple enregistrement de l'information, en passant par des investigations sur les causes et les conséquences, jusqu'aux mesures d'intervention.

\section{APPLICATION PRATIQUE}

Les Recommandations de la Commission traitent de manière tout à fait différente deux conditions bien distinctes d'exposition :

a) celles où l'exposition est prévue et peut être limitée par le contrôle;

b) celles où la source de l'exposition a échappé au contrôle, si bien que toute exposition consécutive ne peut être limitée en importance — si toutefois cela est possible - que par des mesures d'intervention.

Dans le cas de l'exposition à l'irradiation naturelle à des niveaux " normaux ", sans modification due aux activités humaines, on considère que le système de limitation des doses ne s'applique pas.

En cas d'exposition accidentelle, on considère que les limites d'équivalent de dose ne s'appliquent pas. Cependant, la question se pose de savoir quelles sont les mesures d'intervention dont on dispose pour limiter la dose qui en 
résultera. Dans de tels cas, les risques ou les coûts sociaux liés à toute contremesure doivent être justifiés par la réduction de risque qu'elle apporte. En général, le processus d'optimisation est utile et il ne sera bon d'engager des mesures d'intervention que si leur coût social et le risque qu'elles comportent sont inférieurs à ceux qui résulteraient d'une exposition supplémentaire. L'autorité responsable peut, parfois, fixer les niveaux d'intervention auxquels on devra envisager diverses contre-mesures.

L'expression exposition médicale s'applique à l'exposition des personnes soumises à des examens médicaux ou à des traitements impliquant les rayonnements. Les procédures médicales ont pour objet : les examens ou traitements directement associés à une maladie; les examens systématiques de groupe ou les bilans de santé périodiques; les examens pour la surveillance médicale des travailleurs et les examens pratiqués à des fins médico-légales ou d'assurance; les examens ou traitements liés à un programme de recherche médicale. Les principes de justification et d'optimisation sont valables pour toutes les expositions médicales. En général, les individus exposés pour des raisons médicales tirent un bénéfice direct de leur exposition et les limites d'équivalent de dose ne sont pas nécessaires. Toutefois, dans le cas d'irradiation médicale à des fins de recherche médicale, les individus exposés n'en tirent pas de bénéfice direct; il est alors nécessaire de s'assurer que le détriment est acceptable et, donc, de fixer des limites autorisées pour chaque programme de recherche.

L'exposition professionnelle concerne l'exposition des individus au cours de leur travail. Le système complet de limitation des doses s'applique : justification, optimisation et limites d'équivalent de dose. L'échelle et la forme des problèmes posés par la protection des travailleurs contre les rayonnements varient dans de très larges limites et il y a des avantages pratiques à introduire un système de classification des conditions de travail et un système correspondant de classification des lieux de travail.

Les diverses autres contributions à l'exposition du public peuvent être regroupées en quelques grandes catégories. Ce sont : l'exposition aux sources naturelles de rayonnement et aux pratiques qui entraînent un accroissement du niveau de dose dû au fond de rayonnement naturel; l'exposition aux sources de rayonnement utilisées dans l'industrie, la médecine et la recherche; l'exposition due à la dispersion de substances radioactives dans l'environnement; l'exposition consécutive à l'utilisation dans la vie courante de biens de consommation contenant des sources de rayonnement ionisant; l'exposition due à l'utilisation des sources de rayonnement dans l'enseignement. Pour toutes ces catégories, on peut appliquer les principes généraux de justification, d'optimisation et de limitation des doses. En général, les limites dérivées et autorisées sont très utiles, de même que les niveaux d'investigation et d'enregistrement à des fins de surveillance.

\section{CONCLUSION}

Cet exposé forcément schématique et incomplet avait surtout comme intention d'éclairer la signification profonde et l'application pratique du système de limitation des doses de la publication 26. 
Les recommandations précédentes utilisaient surtout l'approche individuelle ou ponctuelle faisant appel aux notions d'organe ou de groupe critiques, afin que la protection des plus exposés assure ipso facto celle de l'ensemble. Les nouvelles recommandations ajoutent l'approche collective ou globale prenant en compte le risque total de l'organisme et l'exposition de l'ensemble des personnes, afin que la protection couvre la totalité du détriment possible.

Les limites de dose anciennes étaient fixées de façon arbitraire ou empirique avec pour principal mérite de remédier à des situations périlleuses en particulier dans le domaine professionnel. Dans sa Publication 26 la Commission a voulu exposer les critères d'acceptabilité des limites de dose, soit absolus pour les effets non stochastiques à seuil, soit relatifs pour les effets stochastiques, par comparaison avec les autres risques communément acceptés dans la vie professionnelle ou publique.

De façon concise, on peut dire que les publications antérieures avaient visé à obtenir une bonne protection radiologique et que les nouvelles recommandations recherchent la protection la meilleure possible. 